\title{
Analgesic effects of intra-articular botulinum toxin Type B in a murine model of chronic degenerative knee arthritis pain
}

This article was published in the following Dove Press journal:

Journal of Pain Research

4 September 2010

Number of times this article has been viewed

\author{
Stephanie Anderson ${ }^{1,2}$ \\ Hollis Krug ${ }^{1,2}$ \\ Christopher Dorman' \\ Pari McGarraugh' \\ Sandra Frizelle' \\ Maren Mahowald ${ }^{1,2}$ \\ 'Rheumatology Section, Veteran's \\ Affairs Medical Center, Minneapolis, \\ Minnesota; ${ }^{2}$ Division of Rheumatology \\ and Autoimmune Diseases, University \\ of Minnesota Medical School, \\ Minneapolis, Minnesota, USA
}

Correspondence: Stephanie R Anderson VA Medical Center, Rheumatology

(I I I-R), One Veteran's Drive, Minneapolis, MN 554I7, USA

Tel + I 6124674190

Fax + I 612 4672267

Email drsranderson@gmail.com
Objective: To evaluate the analgesic effectiveness of intra-articular botulinum toxin Type B (BoNT/B) in a murine model of chronic degenerative arthritis pain.

Methods and materials: Chronic arthritis was produced in adult C57B16 mice by intraarticular injection of Type IV collagenase into the left knee. Following induction of arthritis, the treatment group received intra-articular BoNT/B. Arthritic control groups were treated with intra-articular normal saline or sham injections. Pain behavior testing was performed prior to arthritis, after induction of arthritis, and following treatments. Pain behavior measures included analysis of gait impairment (spontaneous pain behavior) and joint tenderness evaluation (evoked pain response). Strength was measured as ability to grasp and cling.

Results: Visual gait analysis showed significant impairment of gait in arthritic mice that improved $43 \%$ after intra-articular BoNT/B, demonstrating a substantial articular analgesic effect. Joint tenderness, measured with evoked pain response scores, increased with arthritis induction and decreased $49.5 \%$ after intra-articular BoNT/B treatment. No improvement in visual gait scores or decrease in evoked pain response scores were found in the control groups receiving intra-articular normal saline or sham injections. Intra-articular BoNT/B was safe, and no systemic effects or limb weakness was noted.

Conclusions: This study is the first report of intra-articular BoNT/B for analgesia in a murine model of arthritis pain. The results of this study validate prior work using intra-articular neurotoxins in murine models. Our findings show chronic degenerative arthritis pain can be quantitated in a murine model by measuring gait impairment using visual gait analysis scores (spontaneous pain behavior) and joint tenderness scores (evoked pain responses). Reduction of joint pain seen in this study is consistent with our hypothesis of inhibition of release of pain mediators by intra-articular BoNT/B, supporting further investigation of this novel approach to treatment of arthritis pain with intra-articular neurotoxins.

Keywords: intra-articular BoNT/B, osteoarthritis

\section{Introduction}

There is an ongoing need for new therapies for refractory arthritis pain. Osteoarthritisrelated joint pain is a major cause of physical limitation, disability, morbidity, and increased health care utilization for the more than 27 million Americans affected by osteoarthritis. ${ }^{1,2}$ Up to $80 \%$ of patients with osteoarthritis have movement limitation and $25 \%$ cannot perform major activities of daily living. ${ }^{3}$ Up to $20 \%$ of the adult population is affected by chronic knee pain and associated disability, with $11 \%$ of adults with knee osteoarthritis needing help with personal care. Osteoarthritis of the knee is currently one of the five leading causes of disability among noninstitutionalized 
adults. ${ }^{3-5}$ Additionally, $40 \%$ of adults with knee osteoarthritis reported their health as "poor" or "fair". ${ }^{3}$

The primary focus of osteoarthritis care is joint pain management because there are no disease modifying agents available for osteoarthritis. Goals of osteoarthritis treatment include improved pain control and maintenance or improvement of joint function. Currently available systemic analgesics have a significant risk of potentially serious side effects. Insufficient joint pain relief, intolerable drug side effects, and adverse drug interactions are limitations of available oral analgesics. ${ }^{6,7}$ Localized therapies with intra-articular corticosteroids and viscosupplementation are alternative pain control options, but effects and duration may be variable. Minimally invasive surgical intervention for patients failing systemic and local therapies includes arthroscopic lavage and debridement. However, there is increasing evidence that arthroscopic debridement outcomes may be no better than placebo procedures or optimized physical and medical therapy. ${ }^{8,9}$ Joint replacement for severe disabling degenerative arthritis pain carries significant surgical risks, and is often not an option for many patients due to comorbid medical conditions or advanced age. As average life expectancy continues to increase, the burden of disabling degenerative arthritis pain is anticipated to increase as well. Safe, effective, chronic arthritis pain treatment remains an unmet need for many patients, and represents a growing socioeconomic burden in an aging population. ${ }^{10}$

Osteoarthritis is characterized pathologically by bony outgrowths (osteophytes), changes in subchondral and marginal bone, bone marrow edema, and damage to articular cartilage surfaces, leading to loss of joint space and joint misalignment. Soft tissue changes include variable degrees of synovial inflammation, capsular thickening, and ligament laxity. ${ }^{11,12}$ The periosteum and subchondral and marrow bone are richly innervated with sensory fibers, but our current understanding of the cause of arthritis pain remains limited. Most chronic arthritis research and treatment has focused on the degenerative mechanisms and immunologic processes associated with progressive joint damage, rather than the pathogenesis of arthritis-induced pain. Studies of the mechanisms of pain in arthritis have shown that inflammation within joints causes both peripheral and central sensitization of neurons, with spontaneous joint pain at rest and hyperalgesia. ${ }^{13}$ Given this peripheral sensitization, arthritis pain may be treated effectively by intra-articular neurotoxins. Reduction in arthritis pain following use of intra-articular botulinum toxin Type A (BoNT/A) in humans and in murine models of arthritis has been reported. ${ }^{14-17}$ We hypothesized that botulinum toxin Type B (BoNT/B) would also reduce chronic arthritic knee pain. Intra-articular BoNT/B is another option for arthritis pain control that may be superior to other BoNT serotypes. To test this hypothesis, we measured the effect of intra-articular BoNT/B on arthritis pain in a murine model of chronic degenerative arthritis.

\section{Methods}

\section{Animal subjects}

Forty C57B16 mice (Jackson Laboratories, Bar Habor, ME) aged 6-8 weeks old were used in this animal study which was approved by the Minneapolis Veterans Affairs Medical Center Institutional Animal Care and Utilization Committee. The animals were housed in groups of eight animals in the Animal Care and Research Facility at the Minneapolis Veterans Affairs Medical Center, a facility approved by the Association for Assessment and Accreditation of Laboratory Animal Care International. The care and studies of these animals were performed in accordance with the guidelines established in the Guide for the Care and Use of Laboratory Animals (The National Academies Press, USA).

\section{Collagenase-induced chronic degenerative arthritis model}

Chronic arthritis pain was produced in 40 C57B16 mice by intra-articular injection of 10 IU Type IV collagenase (Worthington Biomedical Corporation, Lakeville, NJ) in $10 \mu \mathrm{L}$ normal saline into the left knee. We used a 30 gauge needle with a customized sheath that limited depth of needle penetration to $2.5 \mathrm{~mm}$. The injection was performed through the midline of the patellar tendon just inferior to the patella to ensure accurate entry into the articular space of the knee. Prior to injection, the area was shaved and sterilized with alcohol and animals were anesthetized with isofluorane inhalation. Arthritis was evaluated four weeks after intraarticular collagenase injection. Mice were evaluated for spontaneous pain behavior, evoked pain behavior, and safety using a battery of standardized measures described below. The timepoints for behavioral testing were prior to and after induction of arthritis, and after treatment of arthritis pain.

\section{Spontaneous pain behavior: measurement of gait impairment}

Visual gait analysis was performed by walking the animals on a motorized treadmill (Columbus Instruments, Columbus, $\mathrm{OH})$ at a constant speed of $17 \mathrm{~cm} / \mathrm{sec}$ for a total time of 20 seconds. Gait was evaluated visually and graded 
semiquantitatively on a scale of $0-4$ as a consensus score among three experienced examiners. Gait was defined as normal (4) if the animal was easily able to maintain a consistent speed while walking on the treadmill. Scores of 3, 2, and 1 were given for minimal, moderate, and significant gait impairment, respectively (Table 1).

\section{Evoked pain behavior: measurement of joint tenderness}

Evoked pain behavior (tenderness) was measured by tallying fights (kicks, attempts to break from restraint) and vocalizations for one minute in response to repeated firm palpation of the knee. A single examiner performed all examinations and was blinded as to treatment group assignment. A Palpometer ${ }^{\circledR}$ (Palpometer Systems, Inc., Victoria, BC) was used to train the examiner to apply consistent and precise firm pressure, defined as a level of 4 on the Palpometer $\left(1100 \mathrm{gf} / \mathrm{cm}^{2}=15.6 \mathrm{psi}\right)$. Pressure of this magnitude was high enough to elicit a significant pain response from arthritic joints, but not from normal joints. Both the right (normal) and left knee (arthritic) were examined, with the right knee serving as an internal control. The normal right knee was always examined first. In preliminary experiments with tenderness testing, our group found slightly elevated tenderness scores in the nonarthritic knee when the arthritic knee was examined first.

\section{Safety}

Systemic adverse effects were assessed by observing for anorexia, dehydration, hunched posture, poor grooming, coat changes, or other evidence of poor animal well-being. Given botulinum toxin's known effects of muscle weakness, strength was measured at baseline before induction of arthritis pain, four weeks after intra-articular injection of collagenase (arthritic state), and after intra-articular treatments. Change

Table I Gait impairment criteria

\begin{tabular}{|c|c|}
\hline 0 & Mouse refuses to walk on treadmill \\
\hline I & $\begin{array}{l}\text { Mouse walks up to } 30 \% \text { of the allotted time without touching } \\
\text { dividers, jumping, splaying its legs, or dragging hindquarters; } \\
\text { displays severe inconsistencies in gait }\end{array}$ \\
\hline 2 & $\begin{array}{l}\text { Mouse walks up to } 60 \% \text { of the allotted time without touching } \\
\text { dividers, jumping, splaying its legs, or dragging hindquarters; } \\
\text { displays moderate inconsistencies in gait }\end{array}$ \\
\hline 3 & $\begin{array}{l}\text { Mouse walks up to } 90 \% \text { of the allotted time without touching } \\
\text { dividers, jumping, splaying its legs, or dragging hindquarters; } \\
\text { displays minor inconsistencies in gait }\end{array}$ \\
\hline 4 & $\begin{array}{l}\text { Mouse walks the entire time without touching dividers, } \\
\text { jumping, splaying its legs, or dragging hindquarters; displays } \\
\text { no inconsistencies in gait. }\end{array}$ \\
\hline
\end{tabular}

in muscle strength was measured by the ability to grasp a wire grid against resistance and cling to it while inverted. Grasp ability was tested by applying traction to the animal's tail parallel to the wire grid. Cling ability was tested by inverting the mouse on the wire grid three times with tail held down to wire grid. Both grasp and cling ability were graded on a $0-4$ scale. A score of 0 represented inability to grasp or cling to the wire grid. A score of 4 represented a strong grip against resistance and no instability with inversion (Tables 2 and 3).

\section{Intra-articular neurotoxin and controls}

Four weeks following intra-articular injection of 10 IU collagenase into the left knee, 17 animals were treated with intraarticular BoNT/B (Myobloc ${ }^{\circledR}$, Solstice Neurosciences Inc., South San Francisco, CA) 0.02 IU in $5 \mu \mathrm{L}$ of normal saline into the arthritic left knee. Gait assessment, joint tenderness, and strength examinations were performed three days following intra-articular BoNT/B to allow time for the toxin to take effect. Control groups consisted of arthritic animals treated with either intra-articular normal saline or a sham injection to the left knee at the four-week time point. Seven animals received $5 \mu \mathrm{L}$ or $10 \mu \mathrm{L}$ intra-articular normal saline and eight animals received sham injections. Gait impairment assessment, joint tenderness, and strength examinations were performed three days following intra-articular normal saline or sham injections.

\section{Histologic examination of normal and arthritic knees}

Following conclusion of the study, right (normal) and left (arthritic) knees of representative animals were examined for histologic evidence of degenerative arthritis. The animals were humanely euthanized using $\mathrm{CO}_{2}$ gas and secondary exsanguination. Right and left lower extremities were dissected. Articular specimens were fixed in $10 \%$ buffered formalin for 24 hours and decalcified in $10 \%$ ethylenediamine

Table 2 Grasp impairment criteria

\begin{tabular}{ll}
\hline 0 & Mouse gives no resistance to being pulled across screen; \\
unable to grasp the screen \\
Minimal force needed to pull the mouse across the \\
screen; significant difficulty grasping the screen \\
Moderate force needed to pull the mouse across the \\
screen; moderate difficulty grasping the screen \\
Significant force needed to pull the mouse across the \\
screen; minimal difficulty grasping the screen \\
Full force needed to pull the mouse across the screen; \\
no difficulty grasping the screen.
\end{tabular}


Table 3 Cling impairment criteria

\begin{tabular}{|c|c|}
\hline 0 & Mouse cannot hold on when the screen is tilted vertically \\
\hline I & $\begin{array}{l}\text { Mouse has shown that it can grip the screen when vertical but } \\
\text { falls during every inversion }\end{array}$ \\
\hline 2 & $\begin{array}{l}\text { The mouse falls off the screen during two inversions or falls off } \\
\text { the screen during one inversion and displays instability during } \\
\text { the other two inversions }\end{array}$ \\
\hline 3 & $\begin{array}{l}\text { The mouse falls off the screen once or shows instability during } \\
\text { two inversions }\end{array}$ \\
\hline 4 & The mouse does not display instability with any inversion. \\
\hline
\end{tabular}

tetraacetic acid for two weeks before paraffin embedding. Paraffin-embedded specimens were then sectioned and stained with hematoxilyn and eosin.

\section{Statistical methods}

The unpaired Student's t-test was used to compare groups, ie, prior to induction of arthritis, arthritic, treated, and normal right knee values. Comparisons were made between normal, arthritic, BoNT/B-treated arthritic, and saline-treated arthritic controls using unpaired Student's t-tests. The significance level was selected at a $P$ value of 0.05 .

\section{Results}

\section{Pain behaviors following induction} of arthritis by intra-articular collagenase

Arthritis was successfully induced in 40 mice by intraarticular injection of $10 \mathrm{IU}$ collagenase in $10 \mu \mathrm{L}$ normal saline into the left knee. Animals were examined four weeks after intra-articular collagenase for development of arthritis. Significant alterations in gait due to arthritis pain was demonstrated by decline in visual gait score from 3.50 $(\mathrm{SEM}=0.076)$ to $2.36(\mathrm{SEM}=0.112), P<0.0001$. Evoked pain behavior scores induced by palpation of the painful arthritic knee were increased significantly, from a baseline total score of $1.83(\mathrm{SEM}=0.405)$ to $7.23(\mathrm{SEM}=0.953$, $P<0.0001$, Figure 1).

\section{Effects of intra-articular BoNT/B on pain behavior measures following induction of arthritis}

Seventeen mice with collagenase-induced arthritis pain of the left knee were treated at four weeks with intra-articular injection of $0.02 \mathrm{IU}$ BoNT/B in $5 \mu \mathrm{L}$ normal saline into the affected knee. Animals were examined three days following intra-articular BoNT/B to allow time for appearance of botulinum effects. There were significant improvements in both spontaneous pain behavior measures (visual gait impairment scores) and evoked pain behaviors (joint tenderness to palpation scores). Visual gait analysis score improved by $43 \%(P=0.0419)$, and the evoked pain response score decreased by $49.5 \%(P=0.0134)$ following intra-articular BoNT/B (Figure 1).

\section{Effects of intra-articular normal saline and sham injections following induction of arthritis}

Treatment control groups included seven arthritic mice receiving either 5 or $10 \mu \mathrm{L}$ intra-articular normal saline into the left knee, and eight arthritic mice receiving sham injections into the left knee at four weeks following intraarticular collagenase. Animals were examined three days following either normal saline or sham injections and compared with untreated arthritic animals $(n=40)$. There were no significant changes in spontaneous pain behavior scores or evoked pain behavior scores in these control animals. No significant change in visual gait analysis score was noted following either intra-articular normal saline or sham injections ( $P=0.225$ and $P=0.1921$, respectively). Evoked pain response scores following intra-articular normal saline or sham injections did not change significantly $(P=0.9043$ and $P=0.5355$, respectively, Figure 1).

\section{Normal right knee controls}

Throughout all stages of this study the right knee was a normal, nonarthritic internal control. After induction of arthritis, evoked pain behavior response score in the contralateral knee increased from a baseline mean of $0.83(\mathrm{SEM}=0.208)$ to $2.65(\mathrm{SEM}=0.728 . P=0.0083)$.

\section{Safety}

Grasp and cling strength were measured to monitor safety because of the known effects of muscle weakness caused by botulinum toxins. Strength was measured at baseline, after development of arthritis, and in the post-treatment state. Strength was evaluated by measuring ability to grasp and cling. Forty mice were examined at baseline and four weeks following intra-articular collagenase (arthritic state). Strength assessment showed a significant decline in both measures following induction of arthritis pain, prior to treatment with intra-articular BoNT/B. Grasp scores declined 34\% from a baseline score of $3.83(\mathrm{SEM}=0.120)$ to $2.53(\mathrm{SEM}=0.155$, $P<0.0001)$. Cling scores also declined $34 \%$ from a baseline score of $3.67(\mathrm{SEM}=0.129)$ to $2.43(\mathrm{SEM}=0.168$, $P=0.0003)$. After induction of arthritis and three days following intra-articular BoNT/B into the left knee, grasp scores improved 22\% ( $\mathrm{n}=17, P=0.0704)$, cling scores 
A

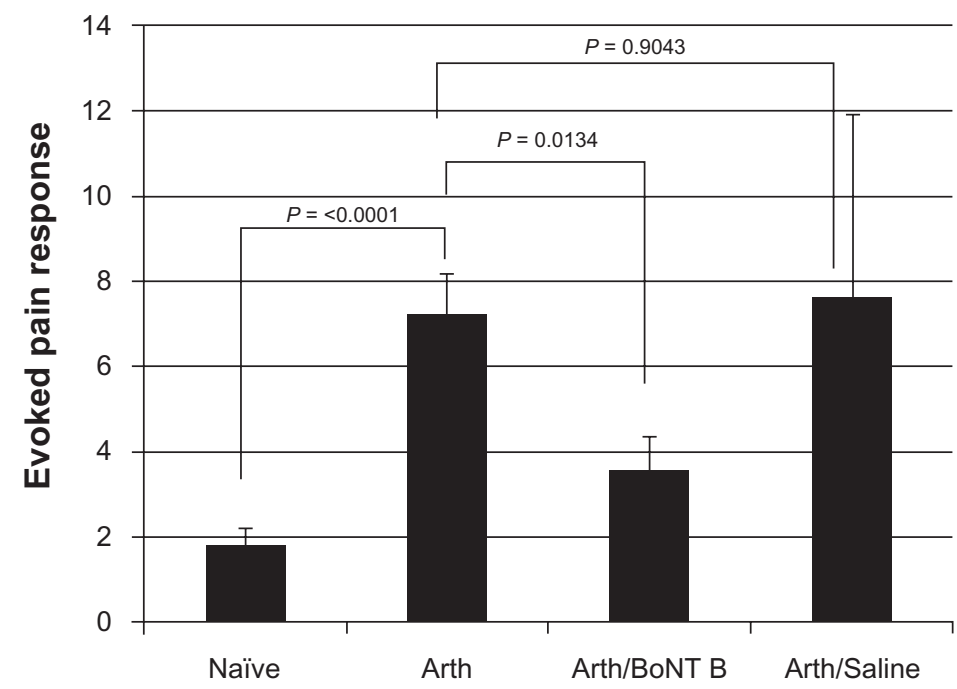

Mean L knee

B

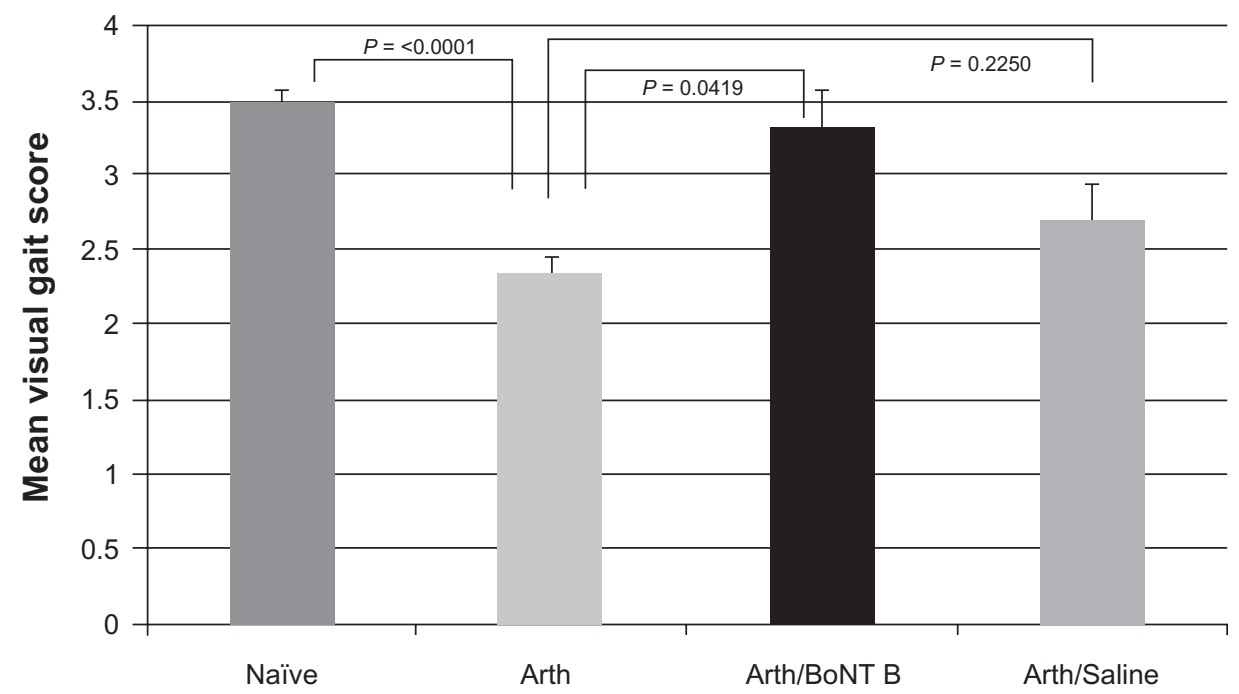

Figures I A) Evoked pain response: Measurement of joint tenderness (left knee) and B) Spontaneous pain behavior: Measurement of gait changes.

Notes: Seventeen mice with collagenase-induced arthritis of the left knee were treated at four weeks with intra-articular botulinum neurotoxin Type B (Myobloc ${ }^{\circledR}$ ) 0.02 IU in $5 \mu \mathrm{L}$ of normal saline in the arthritic left knee. Animals were examined three days following intra-articular botulinum neurotoxin Type $B$ to allow time for botulinum effects. Significant improvements in evoked pain response and spontaneous pain behavior (visual gait analysis) were noted following intra-articular botulinum neurotoxin Type $B$ $(P=0.0134$ and $P=0.0419$, respectively). Gait improved by $43 \%$, and evoked pain response was decreased by $49.5 \%$ following intra-articular botulinum neurotoxin Type B treatment. Treatment control groups included seven arthritic mice receiving either 5 or $10 \mu \mathrm{L}$ intra-articular normal saline in the arthritic left knee at four weeks following intra-articular collagenase. Animals were examined three days following normal saline injections and compared with untreated arthritic animals ( $\mathrm{n}=40$ ). No significant change in evoked pain response or spontaneous pain behavior (visual gait analysis) was noted following intra-articular normal saline $(P=0.9043$ and $P=0.2250$, respectively). Abbreviations: naïve, 40 animals prior to induction of arthritis in the left knee; Arthritic, 40 animals four weeks post intra-articular collagenase into the arthritic left knee; Arth/BoNT/B, 17 animals four weeks post intra-articular collagenase into the left knee (arthritic), three days post intra-articular botulinum neurotoxin Type B into the arthritic left knee; Arth/saline, seven animals four weeks post intra-articular collagenase into the left knee (arthritic), three days post intra-articular saline into the arthritic left knee.

improved $23 \%(\mathrm{n}=17, P=0.2752)$, although not reaching statistical significance in this small study. The seven animals that received intra-articular normal saline injections had no significant change in grasp or cling scores compared with the arthritic state $(P=0.3964$ and $P=0.7457$ respectively, Figure 2). Eight animals that received sham injections had no change in grasp $(P=0.5637)$, but did have a significant decrease in ability to cling $(P=0.0019)$. No signs of anorexia, dehydration, hunched posture, poor grooming, coat changes, or other evidence of poor animal well-being were noted in any animals at any point during the study.

\section{Histologic examination of normal and arthritic knees}

Following conclusion of the study, right (normal) and left (arthritic) knees of representative animals were examined for 


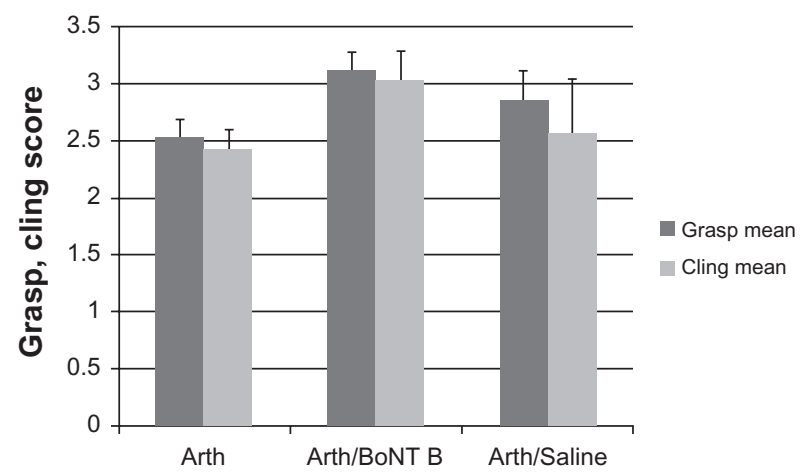

Figure 2 Safety assessment: Limb strength.

Notes: The 17 animals receiving intra-articular botulinum neurotoxin Type B were assessed for changes in strength three days following intra-articular botulinum neurotoxin Type B. No significant change in grasp or cling scores were noted following intra-articular botulinum neurotoxin Type B compared with untreated arthritic animals $(P=0.0704$ and $P=0.2752$, respectively). The seven animals receiving intra-articular normal saline injections had no significant change in grasp or cling scores $(P=0.3964, P=0.7457$, respectively)

Abbreviations: arthritic, 40 animals four weeks post intra-articular collagenase into the arthritic left knee; Arth/BoNT B, 17 animals four weeks post intra-articular collagenase into the left knee (arthritic), three days post intra-articular botulinum neurotoxin Type B into the arthritic left knee; Arth/saline, seven animals four weeks post intra-articular collagenase into the left knee (arthritic), three days post intraarticular saline into the arthritic left knee.

histologic evidence of degenerative arthritis. Hematoxilyn and eosin staining of knees revealed irregularities and thinning of articular cartilage and early osteophyte formation compared with normal knees, ie demonstrating changes consistent with osteoarthritis (Figures 3 and 4).

\section{Discussion}

This study is the first report of intra-articular BoNT/B for analgesia in a murine model of arthritis pain. The results of this study validate prior work using intra-articular neurotoxins in murine models. ${ }^{16}$ Our findings show chronic degenerative arthritis pain can be quantified in a murine model by measuring gait impairment with visual gait analysis scores (ie, spontaneous pain behavior) and joint tenderness scores (ie, evoked pain responses). Visual gait analysis showed significant impairment of gait in arthritic mice that improved $43 \%$ after intra-articular BoNT/B, demonstrating a substantial articular analgesic effect. Joint inflammation is not a prominent feature of degenerative arthritis, but joint tenderness, measured with evoked pain response scores, increased with arthritis induction and decreased $49.5 \%$ after intra-articular BoNT/B treatment. These changes in joint tenderness were clinically and statistically significant even with the relatively small animal numbers used in this study. Reduction of joint pain seen in this study is consistent with our hypothesis of inhibition of release of pain mediators by intra-articular BoNT/B.

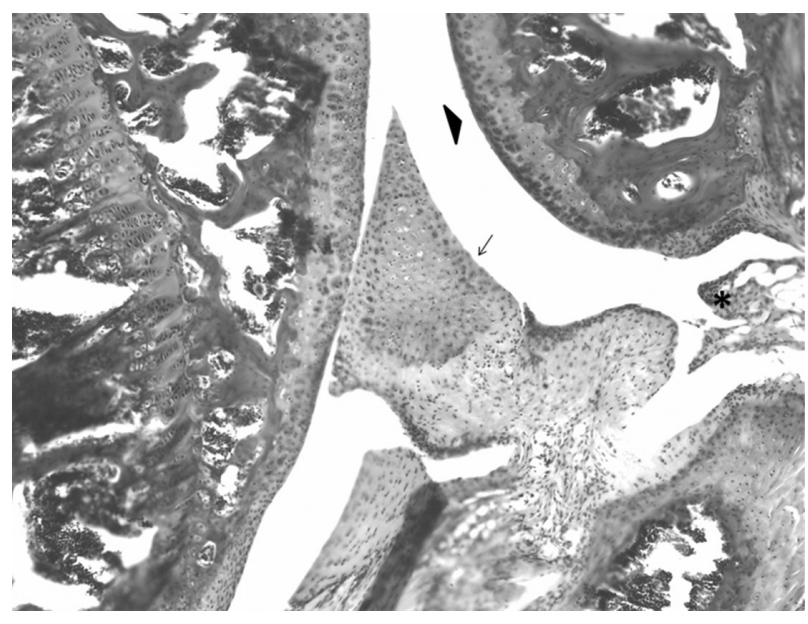

Figure 3 Hematoxylin and eosin stained normal right knee.

Note: Normal-appearing articular cartilage (arrowhead), meniscus (arrow), and synovium (asterisk).

There was a small increase in the evoked pain behavior response score in the contralateral nonarthritic right knee that was not clinically significant. This interesting pain response in the normal contralateral limb is similar to findings noted by other groups studying monoarthritis in murine models. Lam et al reported that substance $\mathrm{P}$ exacerbated and spread the early signs of disease, such as increased blood flow and vascular permeability, to contralateral joints. ${ }^{18}$

\section{Role for intra-articular botulinum therapy}

Botulinum neurotoxin (BoNT) is produced by Clostridium botulinum as a complex of proteins containing the neurotoxic

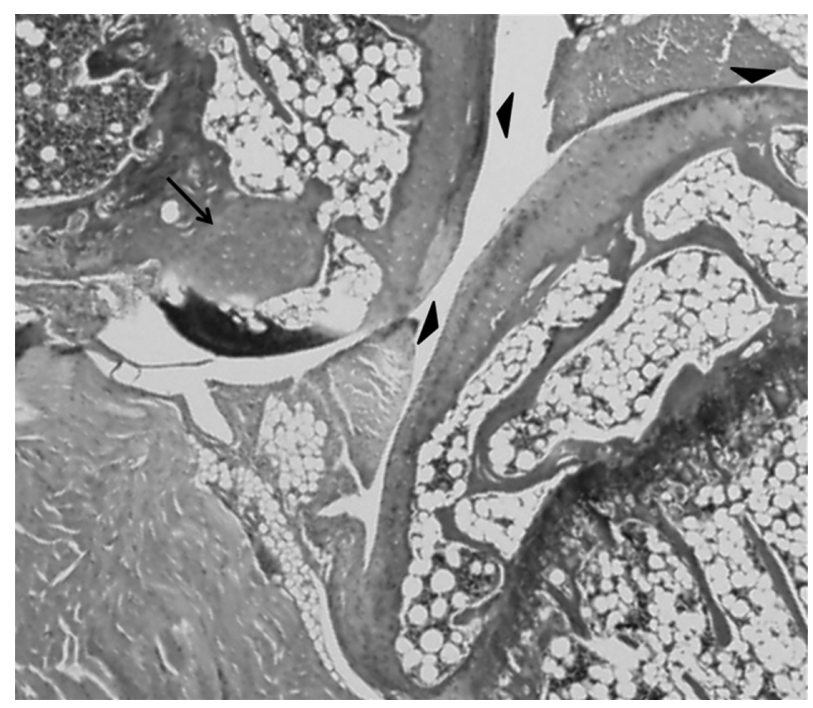

Figure 4 Arthritic left knee stained with hematoxylin and eosin.

Note: Irregularities and thinning of articular cartilage (arrowheads) and early osteophyte formation (arrow) consistent with degenerative arthritis. 
moiety associated with nontoxic components. There are seven serologically distinct BoNT serotypes that all act by inhibiting release of signal chemicals packaged in neuronal vesicles. ${ }^{19}$ The exocytosis of neuronal signal chemicals is dependent on the function of the N-ethylmaleimide-sensitive factor attachment protein receptor complex, collectively called the soluble $\mathrm{N}$-ethylmaleimide-sensitive factor activating protein receptor (SNARE) proteins ${ }^{19,20}$ All serotypes of BoNT cleave SNARE proteins. The specific target site within the SNARE complex is dependent on the BoNT serotype. ${ }^{19}$ Although all BoNTs act by disabling SNARE-associated exocytosis, the potencies and characteristics of their actions vary. ${ }^{19-21}$ Botulinum toxins affect striated muscle by creating a chemical denervation that is temporary and reversible through highly potent inhibition of acetylcholine release at the neuromuscular junction. ${ }^{20}$ Acetylcholine is not the only neurotransmitter affected by BoNTs. BoNTs have also been found to affect the release of multiple SNARE-dependent neuropeptides, including substance P, glutamate, and calcitonin gene-related peptide, all important mediators of articular pain transmission. ${ }^{15,22}$

BoNTs are the most potent neurotoxins known. However, small doses are successful as cosmetic and musculoskeletal therapies. Currently, BoNT/A and BoNT/B are the best characterized and most used clinically. BoNT/A injections are analgesic for painful muscle contractions associated with cervical dystonia, migraine/tension headaches, and myofascial pain syndromes. ${ }^{23}$ In BoNT/A treatment of painful soft tissue syndromes, pain relief preceded the resolution of muscle contractions, suggesting that BoNTs may have antinociceptive effects independent of known effects on neuromuscular junctions. ${ }^{24}$ BoNT/A inhibited capsaicinstimulated release of substance $\mathrm{P}$ from embryonic rat dorsal root ganglia neurons in culture. ${ }^{25}$ Subcutaneous BoNT/A paw injections in a formalin-induced rat model of pain reduced electrical excitations and c-fos expression in the spinal cord, and reduced edema and tissue glutamate release. ${ }^{26}$

Efficacy of intra-articular BoNT/A for refractory arthritis pain in humans, and in murine models of arthritis joint pain, has been reported recently. Intra-articular BoNT/A reduced lower extremity arthritis pain by an average of $55 \%$, and shoulder pain by an average of $71 \%$ in a study of 11 patients with chronic arthritis pain refractory to intra-articular corticosteroids, with no noted adverse effects. ${ }^{14}$ In another study of patients with refractory axial skeletal pain, eight of 11 reported a decrease in pain score, improved activities of daily living, and range of motion following BoNT injections. These BoNT injections for axial skeletal pain provided longer lasting pain relief than corticosteroid injections. ${ }^{27}$ Similar results were found in a randomized placebo-controlled trial of BoNT/A in chronic severe shoulder pain. ${ }^{17}$ Another randomized controlled trial found that intra-articular BoNT/A was as effective as intra-articular corticosteroids for chronic knee pain. ${ }^{28}$ Krug et al have reported significant analgesic effects of intra-articular BoNT/A in murine models of chronic inflammatory arthritis. ${ }^{16}$ Analgesic effects were not found in the acute carrageenan arthritis pain model.

Intra-articular BoNT/B was safe, with no weakness of limb muscles or systemic effects noted. This finding of safety confirms our prior work with intra-articular BoNT/A in murine arthritis models and small human studies. It is possible that higher doses of intra-articular BoNT/B could be used to optimize analgesic effects. The current study does have the noted weakness that our visual gait analysis system may not be sensitive enough to measure full treatment effect, therefore future studies will incorporate computerized digital gait analysis. Additionally, in this preliminary study, the duration of action of intra-articular BoNT/B has not been fully explored. Evaluation of dose response and duration of effect are future research directions for our group. Mixtures of various intra-articular botulinum serotypes may provide faster onset of action and longer duration of effects. Such mixtures may prove useful in other types of articular pain.

This study supports the hypothesis that chronic arthritis pain may be amplified by neuropeptide release in the periphery. Inhibition of neuropeptide release may have altered nociceptor function, and reduced pain generation and neurogenic inflammation. This selective chemodenervation of articular pain fibers with intra-articular injection of neurotoxins is a novel local approach to treatment of arthritis joint pain. Interruption of neuropeptide release by intra-articular BoNT/B appeared to decrease pain responses in the joint and improve gait abnormalities. The results of this study support further investigation of this novel approach to treatment of arthritis pain with intra-articular neurotoxins.

\section{Disclosure}

The authors have no conflicts of interest in this work.

\section{References}

1. Hochberg MC. Multidisciplinary integrative approach to treating knee pain in patients with osteoarthritis. Ann Intern Med. 2003;139: 724-730.

2. Lawrence RC, Felson DT, Helmick CG, et al. Estimates of the prevalence of arthritis and other rheumatic conditions in the United States. Part II. Arthritis Rheum. 2008;58:26-35.

3. CDC.gov. Division of Adult and Community Health, National Center for Chronic Disease Prevention and Health Promotion, Osteoarthritis 2009 Aug 1 [updated 2010 Feb 4]. Available at: http://www.cdc.gov/ arthritis/basics/osteoarthritis.htm. Accessed May 23, 2010. 
4. McAlindon T, Cooper C, Kirwan J, Dieppe P. Knee pain and disability in the community. Br J Rheumatol. 1992;23:189-192.

5. Ettinger W, Davis M, Neurhaus J, Mallon K. Long-term physical functioning in persons with knee osteoarthritis from NHANES I: Effects of comorbid medical conditions. J Clin Epidemiol. 1994;47: 809-815.

6. Mahowald M. The role of nonprescription analgesics in treating mild to moderate pain. Clinical and economic considerations. Minneapolis Health Learning Systems. 2000.

7. Mahowald M. Chronic pain management. In: Ruddy S, Harris E, Sledge C, Budd R, Sergent J, editors. Kelley's Textbook of Rheumatology. Philadelphia, PA: WB Saunders; 2004.

8. Moseley JB, O'Malley K, Petersen NJ, et al. A controlled trial of arthroscopic surgery for osteoarthritis of the knee. N Engl J Med. 2002; 347:81-88.

9. Kirkley A, Birmingham TB, Litchfield RB, et al. A randomized trial of arthroscopic surgery for osteoarthritis of the knee. $N$ Engl J Med. 2008;359:1097-1107.

10. Curatolo M, Bogduk N. Pharmacologic pain treatment of musculoskeletal disorders: Current perspectives and future prospects. Clin J Pain. 2001;17:25-32.

11. Kidd BL. Osteoarthritis and joint pain. Pain. 2006;123:6-9.

12. Felson DT, Lawrence RC, Dieppe PA, et al. Osteoarthritis: New insights. Part 1: The disease and its risk factors. Ann Intern Med. 2000; 133:635-646.

13. Schaible HG, Schmelz M, Tegeder I. Pathophysiology and treatment of pain in joint disease. Adv Drug Deliv Rev. 2006;58:323-342.

14. Mahowald ML, Singh JA, Dykstra D. Long term effects of intraarticular botulinum Type A for refractory joint pain. Neurotox Res. 2006;9:179-188.

15. Mahowald ML, Krug HE, Singh JA, et al. Intra-articular botulinum toxin type A: A new approach to treat arthritis joint pain. Toxicon. 2009; $54: 658-667$
16. Krug HE, Frizelle S, McGarraugh $\mathrm{P}$, et al. Pain behavior measures to quantitate joint pain and response to neurotoxin treatment in murine models of arthritis. Pain Med. 2009;10:1218-1228.

17. Singh JA, Mahowald ML, Noorbaloochi S. Intra-articular botulinum toxin A for refractory shoulder pain: A randomized, double-blinded, placebo-controlled trial. Transl Res. 2009;153:205-216.

18. Lam FF, Wong HH, Ng ES. Time course and substance P effects on the vascular and morphological changes in adjuvant-induced monoarthritic rats. Int Immunopharmacol. 2004;4:299-310.

19. Dolly JO, Aoki KR. The structure and mode of action of different botulinum toxins. Eur J Neurol. 2006;13 Suppl 4:1-9.

20. Flynn TC. Myobloc. Dermatol Clin. 2004;22:207-211.

21. Aoki KR, Guyer B. Botulinum toxin type A and other botulinum serotypes. Eur J Neurol. 2001:8 Suppl 5:21-29.

22. Aoki KR. Botulinum toxin: A successful therapeutic protein. Curr Med Chem. 2004;11:3085-3092.

23. Smith HS, Audette J, Royal MA. Botulinum toxin in pain management of soft tissue syndromes. Clin J Pain. 2002;6 Suppl:S147-S154.

24. Aoki KR. Evidence for antinociceptive activity of botulinum toxin Type A in pain management. Headache. 2003;43 Suppl 1:S9-S15.

25. Purkiss J, Welch M, Doward S, et al. Capsaicin stimulated release of substance $P$ from cultured dorsal root ganglion neurons involvement of two distinct mechanisms. Biochem Pharmacol. 2000;59:1403-1406.

26. Cui M, Li Z, You S, et al. Mechanisms of the antinociceptive effect of subcutaneous Botox: Inhibition of peripheral and central nociceptive processing. Arch Pharmacol. 2002;365:R17.

27. Dykstra D. The effects of intra-articular botulinum toxin on sacroiliac, cervical/lumbar facet and sternoclavicular joint pain and C-2 root and lumbar disc pain: A case series of 11 patients. The Pain Clinic. 2007; 19:27-32.

28. Boon AJ, Smith J, Dahm DL, et al. Efficacy of intra-articular botulinum toxin type A in painful knee osteoarthritis: A pilot study. $P M R$. 2010; 2:268-276.
Journal of Pain Research

\section{Publish your work in this journal}

The Journal of Pain Research is an international, peer-reviewed, open access, online journal that welcomes laboratory and clinical findings in the fields of pain research and the prevention and management of pain. Original research, reviews, symposium reports, hypothesis formation and commentaries are all considered for publication.

\section{Dovepress}

The manuscript management system is completely online and includes a very quick and fair peer-review system, which is all easy to use. Visit http://www.dovepress.com/testimonials.php to read real quotes from published authors. 